\title{
Computing Interpolants without Proofs ${ }^{\star}$
}

\author{
Hana Chockler, Alexander Ivrii, Arie Matsliah \\ IBM Research - Haifa
}

\begin{abstract}
We describe an incremental algorithm for computing interpolants for a pair $\varphi_{A}, \varphi_{B}$ of formulas in propositional logic. In contrast with the common approaches, our method does not require a proof of unsatisfiability of $\varphi_{A} \wedge \varphi_{B}$, and can be realized using any SAT solver as a black box. We achieve this by combining model enumeration with the ability to easily generate interpolants in the special case that one of the formulas is a cube.
\end{abstract}

\section{Introduction}

Craig's interpolation theorem [Cra57] states that for any pair of propositional formulas $\varphi_{A}, \varphi_{B}$, if $\varphi_{A}$ implies $\neg \varphi_{B}\left(\varphi_{A} \Rightarrow \neg \varphi_{B}\right)$ then there exists a formula $\varphi_{I}$, so that $\varphi_{A} \Rightarrow \varphi_{I} \Rightarrow \neg \varphi_{B}$, and in $\operatorname{addition} \operatorname{Vars}\left(\varphi_{I}\right) \subseteq$ $\operatorname{Vars}\left(\varphi_{A}\right) \cap \operatorname{Vars}\left(\varphi_{B}\right)$. The formula $\varphi_{I}$ is called a Craig interpolant of $\varphi_{A}$ and $\varphi_{B}$.

Starting with the seminal work of McMillan [McM03], interpolants have a central role in formal verification (and beyond) - various application include hardware model checking [McM03,McM05], detection of functional dependency [LJHM07], Boolean function decomposition [LJH08], and model checking of sequential programs [McM10].

The most common technique for computing an interpolant for a pair of formulas $\varphi_{A}, \varphi_{B}$ in propositional logic is based on a resolution refutation for $\left(\varphi_{A} \wedge \varphi_{B}\right)$ produced by a DPLL-like SAT solver [ZM03,ANORC10]. Once obtained, the proof can be transformed into an interpolant in the form of a Boolean circuit having the same structure as the proof itself [Kra97,Pud97,McM03,KW10]. ${ }^{1}$

Even though this scheme is generally very successful in practice, its main limitation is the need for a refutation (proof of unsatisfiability) that is of manageable size. Since modern SAT solvers are not specifically aimed

\footnotetext{
* This work is partially supported by the European Community under the call FP7ICT-2009-5 - project PINCETTE 257647.

${ }^{1}$ There are efficient algorithms known to compute interpolants based on refutations in proof systems other than resolution (e.g., in Cutting Planes [Kra97]), but the one based on resolution is the canonical one from practical perspective.
} 
to produce short refutations, even for simple problems the interpolants produced are often too big to handle. In addition, the interpolants constructed in this way are usually highly redundant, and for practical applications it is often beneficial to minimize/simplify them. However, such minimization can be very costly - and thus in practice one might not succeed to construct a small interpolant even if one exists. In the worst case, the input formula $\left(\varphi_{A} \wedge \varphi_{B}\right)$ might not have a resolution refutation of polynomial (in the number of variables) size at all.

The problem boils down to the dependency of the method on a particular type of SAT solving algorithm. In other words, even if the ultimate SAT solver was given to us as an oracle that answers any satisfiability query instantly, we would not know how to use it to produce interpolants efficiently.

\subsection{Our contribution}

In this paper we present a SAT-based incremental algorithm for computing interpolants. Our algorithm can be easily implemented on top of any complete SAT-solver with the minimal interface to return SAT / UNSAT and a satisfying assignment in case of $S A T$, and in particular the solver does not need to produce proofs or be DPLL-based. Even though this minimal interface in principle suffices, for practical reasons we will want from the solver a bit more: the (now standard) incremental interface that allows adding clauses between calls and solving under assumptions (see Section 3).

The core idea of our algorithm is to compute an interpolant $\varphi_{I}$ incrementally by taking the disjunction of "point" interpolants $\varphi_{p}$ for all $p \in A$ (where $A$ denotes the set of models of $\varphi_{A}$ ). Each point interpolant $\varphi_{p}$ contains $p$, is disjoint from $B$, and is defined in terms of common variables of $\varphi_{A}$ and $\varphi_{B}$. It follows that the union of these interpolants for all $p \in A$ contains all of $A$ and is still disjoint from $B$ - thus constituting a valid interpolant for $\left(\varphi_{A}, \varphi_{B}\right)$. An important observation here is that computing a point interpolant, or more generally an interpolant between a cube $c=\ell_{1} \wedge \cdots \wedge \ell_{k}$ (containing up to $2^{-k}$ fraction of the points in $A)$ and any propositional formula $\varphi_{B}$ is trivial: one can simply remove from $c$ the literals referring to variables local to $A$. From the practical viewpoint, it is crucial to further generalize these cubes to cover as many points of $A$ as possible while still being disjoint from $B$. The difference between our approach and the traditional (monolithic) interpolation is depicted graphically in Figure 1. 


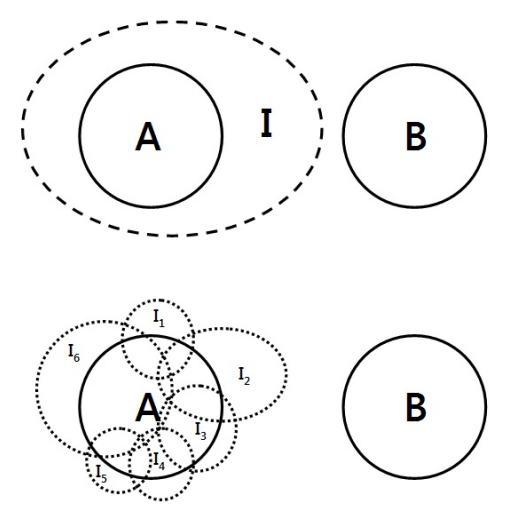

Fig. 1. Comparison between monolithic and incremental interpolants

A more general method of computing an interpolant for $\left(\varphi_{A}, \varphi_{B}\right)$ is the following two-stage process. First, we show that $\varphi_{A} \wedge \varphi_{B}$ is unsatisfiable by means of a certain partition-based algorithm (by this we mean an algorithm in which one solver is run on $\varphi_{A}$, another solver is run on $\varphi_{B}$, and the two solvers are allowed to exchange constraints consisting only of common variables). Second, we show how to construct an interpolant based on these exchanged constraints (see Section 3.3 for details).

Our approach is somewhat similar in spirit to the classic algorithm that extracts a satisfying assignment from a SAT decision procedure, viewing the procedure as an oracle. In this algorithm, the variables are ordered, and then the first one is assigned at random. The algorithm then queries the SAT oracle for the existence of a satisfying assignment for the rest of the formula; it continues in this way until all variables are assigned.

\subsection{Related Work}

This work is tightly related to various methods for finding all models (satisfying assignments) of a given formula, or more specifically finding all assignments to the common variables of $\varphi_{A}$ and $\varphi_{B}$ possessing extensions satisfying $\varphi_{A}$. We refer to the papers [McM02,JS05,BKK11,GM12] containing efficient algorithms for this task and references to earlier work. In particular we also follow the widely used blocking clause approach to prevent the algorithm from discovering the same point again and again, and we try to generalize cubes as much as possible to get quick coverage of $A$ (the set of models of $\varphi_{A}$ ). However, our setting allows an additional twist on the generalization process which makes convergence of interpolant computation quicker than that of computing all satisfying as- 
signments: we can additionally generalize each cube as long as it remains disjoint from $B$, allowing wider coverage of points in $A$.

An alternative partition-based algorithm for detecting whether $\varphi_{A} \wedge$ $\varphi_{B}$ is unsatisfiable appears in [PG00]. In the cited work, all the assignments to the common variables are checked, and $\varphi_{A} \wedge \varphi_{B}$ is unsatisfiable if and only if for every such assignment $c$ either $c \wedge \varphi_{A}$ or $c \wedge \varphi_{B}$ is unsatisfiable. In contrast, our algorithm only considers those assignments to the common variables which admit a satisfiable extension for $\varphi_{A}$ (and in practice this number is much smaller due to generalization).

Last but not least, an important source of inspiration for this work are the papers [BKK11,Bra11] which demonstrate the general power of an incremental approach for solving difficult problems. Note that in the context of model checking, IC3 (the recent breakthrough model-checking technique by Bradley [Bra11]) can be also viewed as a method which generates interpolants without proofs. However our setting is more general, allowing to compute an interpolant for any pair of propositional formulas.

\section{Preliminaries}

As usual, a literal is either a variable or its negation, a clause is a disjunction of literals, and a cube is a conjunction of literals. A CNF is a conjunction of clauses and a $D N F$ is a disjunction of cubes.

Given a (propositional) formula $\varphi_{A}$, we denote by $V_{A}$ the set of all variables that occur in $\varphi_{A}$. Given two formulas $\varphi_{A}$ and $\varphi_{B}$, we denote by $V_{A \cap B} \triangleq V_{A} \cap V_{B}$ the set of common variables of $\varphi_{A}$ and $\varphi_{B}$, and by $V_{A \backslash B}=V_{A} \backslash V_{B}$ the set of all variables local to $\varphi_{A}$.

With each formula $\varphi_{A}$ we associate a subset of $\{0,1\}^{V_{A}}$ containing all assignments to $V_{A}$ satisfying $\varphi_{A}$ (models of $\varphi_{A}$ ). Slightly abusing notation, we sometimes refer to formulas as subsets and vice versa; in particular, $\varphi_{A}=\emptyset$ means that $\varphi_{A}$ is unsatisfiable.

Definition 1 (Interpolant). ${ }^{2}$ Let $\varphi_{A}, \varphi_{B}$ be a pair of formulas that cannot be simultaneously satisfied $\left(\varphi_{A} \wedge \varphi_{B}=\emptyset\right)$. An interpolant $\varphi_{I}=$ $\operatorname{Itp}\left(\varphi_{A}, \varphi_{B}\right)$ of $\left(\varphi_{A}, \varphi_{B}\right)$ is a formula satisfying: 1) $\left.\varphi_{A} \Rightarrow \varphi_{I} ; 2\right) \varphi_{I} \wedge \varphi_{B}=$ $\emptyset$; and 3) $V_{I} \subseteq V_{A \cap B}$.

Our algorithm makes use of a SAT solver to decide satisfiability of a propositional formula. For most of the paper this solver is viewed as a

\footnotetext{
${ }^{2}$ This definition slightly deviates from the original definition of Craig, but is now
} standard in the context of formal methods. 
black box; the only requirements are that it should output SAT or UNSAT depending on the status of the formula, and in the case of $S A T$ the solver should also return an assignment (model) satisfying the formula. Note that a model is a conjunction of unit clauses, or simply a cube.

We denote by $P_{A \cap B}^{A}$ the projection of the set (of all assignments satisfying) $\varphi_{A}$ to $V_{A \cap B} . P_{A \cap B}^{B}$ is defined similarly. Clearly, $\varphi_{A} \wedge \varphi_{B}=\emptyset$ if and only if $P_{A \cap B}^{A} \cap P_{A \cap B}^{B}=\emptyset$.

\section{Algorithm}

In this section we present the main contribution of this paper - an incremental algorithm for computing an interpolant $\varphi_{I}$ of $\left(\varphi_{A}, \varphi_{B}\right)$. In the following subsections we describe a basic version of the algorithm, an efficient implementation of this algorithm on top of a modern CDCL solver (e.g. MiniSat), and various extensions and optimizations which seem crucial for real-life instances.

\subsection{Basic Algorithm}

The algorithm (described in Algorithm 1) accepts a pair $\left(\varphi_{A}, \varphi_{B}\right)$ of propositional formulas, and, in the case under consideration that $\varphi_{A} \wedge \varphi_{B}$ is unsatisfiable, returns an interpolant $\varphi_{I}$ (in DNF). The algorithm also detects the case that $\varphi_{A} \wedge \varphi_{B}$ is satisfiable, and can in principle return a satisfying assignment to $\varphi_{A} \wedge \varphi_{B}$.

The interpolant $\varphi_{I}$ is constructed incrementally; initially it is empty. Roughly speaking, the algorithm searches for points $p \in \varphi_{A}$ not yet covered by $\varphi_{I}$ and then generalizes these points to cubes by omitting assignments to all variables local to $\varphi_{A}$ and to as many common variables as possible, while still keeping these cubes disjoint from $\varphi_{B}$. Each such generalized cube represents a new incremental knowledge and is added to $\varphi_{I}$. For convenience we introduce the set $\varphi_{A}^{\prime}=\varphi_{A} \wedge \neg \varphi_{I}$ corresponding to the set of assignments in $\varphi_{A}$ not yet covered by $\varphi_{I}$; the algorithm terminates when $\varphi_{A}^{\prime}$ becomes empty.

We now describe a single iteration of the main loop (lines 4-17) in detail. On line 4 we call a SAT solver to check whether $\varphi_{A}^{\prime}$ is empty. If so, then $\varphi_{A} \subseteq \varphi_{I}$ and the algorithm terminates providing $\varphi_{I}$ as the final interpolant. Otherwise (line 7), the SAT solver returns a model $p$ for $\varphi_{A}^{\prime}$, that is an assignment to variables in $V_{A}$. First we project this assignment to $V_{A \cap B}$ by omitting the variables local to $\varphi_{A}$ (line 8 ); this projection corresponds to the cube $p^{\prime}$. 


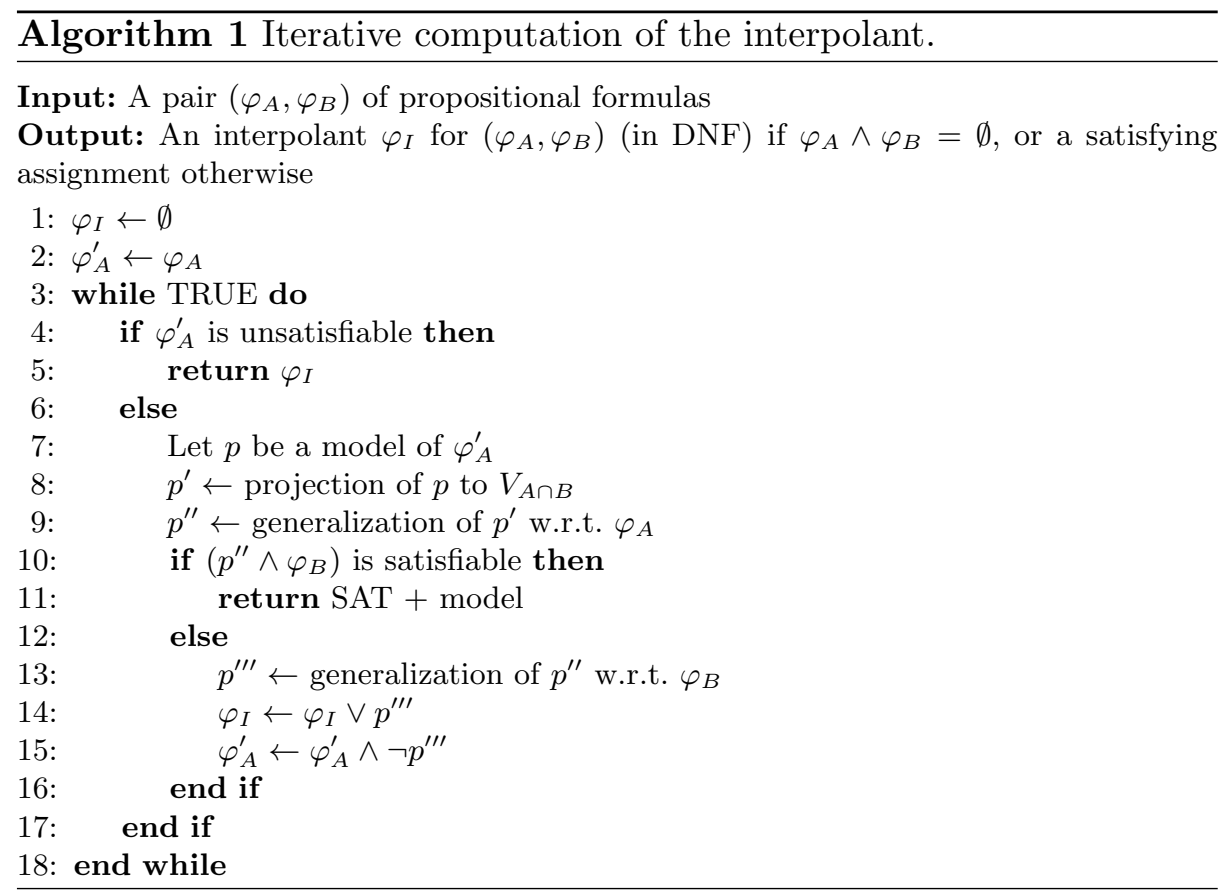

We can (on line 9) further generalize the cube $p^{\prime}$ to $p^{\prime \prime}$ as long as it satisfies the following property: for any extension $\tilde{p}^{\prime}$ of $p^{\prime \prime}$ to $V_{A \cap B}$ there is a further extension $\tilde{p}$ of $\tilde{p}^{\prime}$ to $V_{A}$ which satisfies $\varphi_{A}$. In other words, consider the projection $P_{A \cap B}^{A}$ of all assignments satisfying $\varphi_{A}$ to $V_{A \cap B}$. By construction, $p^{\prime} \in P_{A \cap B}^{A}$, and we seek to generalize it to $p^{\prime \prime}$ so that as sets of assignments $p^{\prime} \in p^{\prime \prime} \subseteq P_{A \cap B}^{A}$, thus enumerating more than one (projection of a) satisfying assignment to $\varphi_{A}$ to $V_{A \cap B}$ at once. We describe the existing methods for such a generalization in Section 3.3. Also note that this generalization is performed with respect to the original set $\varphi_{A}$.

Next (on line 10), we make another call to a SAT solver to check whether $\varphi_{B} \wedge p^{\prime \prime}$ is satisfiable (note that the cube $p^{\prime \prime}$ can be passed to the solver as a set of unit assumptions). If this is the case, then $\varphi_{A} \wedge \varphi_{B}$ is satisfiable, and in fact we can obtain an explicit satisfying assignment to $\varphi_{A} \wedge \varphi_{B}$ by extending the assignment satisfying $p^{\prime \prime} \wedge \varphi_{B}$ to $V_{A \backslash B}$ which satisfies $\varphi_{A}$ (which is possible by the property above). If the generalization step on line 9 is omitted, a satisfying assignment to $\varphi_{A} \wedge \varphi_{B}$ can be obtained immediately by unifying the assignment to $p^{\prime \prime} \wedge \varphi_{B}$ and the assignment $p$ (since the two assignments match on the common variables).

In the main case under consideration, $p^{\prime \prime} \wedge \varphi_{B}$ is unsatisfiable, and we seek (on line 13) to generalize $p^{\prime \prime}$ even further to $p^{\prime \prime \prime}$ by dropping the 
assignments to some of the variables in $V_{A \cap B}$ while keeping $p^{\prime \prime \prime}$ disjoint from $\varphi_{B}$. The difference between this generalization and the one on line 9 is that now we can let $p^{\prime \prime \prime}$ represent non- $P_{A \cap B}^{A}$ points provided that they are also non- $P_{A \cap B}^{B}$ points (see definitions above). In particular, $p^{\prime \prime \prime}$ can also describe additional $P_{A \cap B}^{A}$-points, not previously described by $p^{\prime \prime}$. From the practical viewpoint, this is a very important optimization (details follow).

Note that we can view $p^{\prime \prime \prime}$ as an interpolant of $p$ and $\varphi_{B}$. We update $\varphi_{I} \leftarrow \varphi_{I} \vee p^{\prime \prime \prime}$ (thus keeping $\varphi_{I}$ in DNF) and prevent the solver from rediscovering points in $p^{\prime \prime \prime} \wedge \varphi_{A}$ (and in particular $p$ ) by adding the blocking clause $\neg p^{\prime \prime \prime}$ to $\varphi_{A}^{\prime}$.

Claim 1 (1) Algorithm 1 always terminates. (2) If $\varphi_{A} \wedge \varphi_{B}=\emptyset$ it outputs a valid interpolant $\varphi_{I}$ for $\left(\varphi_{A}, \varphi_{B}\right)$.

Proof. (1) Initially $\varphi_{A}^{\prime}=\varphi_{A}$, and in each iteration its size (as set of assignments) shrinks by $\geq 1$. (2) By construction, $V_{I} \subseteq V_{A \cap B}$. In addition, $\varphi_{I}$ is a disjunction of cubes that are disjoint from $\varphi_{B}$, hence $\varphi_{I} \wedge B=\emptyset$. To see that $\varphi_{A} \Rightarrow \varphi_{I}$, observe that the algorithm terminates only when $\varphi_{A}^{\prime} \triangleq \varphi_{I} \backslash \varphi_{A}$ becomes empty.

\subsection{Implementation Details}

Now we describe how the algorithm proposed in the last section can be efficiently implemented on top of MiniSat or any other SAT solver that provides an interface to incrementally add new clauses into the solver, and to solve under a set of additional unit assumptions [ES03]. In the case of a satisfiable result, such a solver should return a model satisfying all of the clauses in the solver as well as all of the unit assumption literals. In the case of an unsatisfiable result, the solver should return a subset of the assumptions used in the proof of unsatisfiability.

We keep two instances of the SAT solver: $A$-solver holding the CNF for $\varphi_{A}^{\prime}$ and $B$-solver holding the CNF for $\varphi_{B}$. Thus the SAT call on line 4 corresponds to A.Solve() and the SAT call on line 10 corresponds to B.Solve $\left(p^{\prime \prime}\right)$ with the cube $p^{\prime \prime}$ passed as the set of unit assumptions. In the simplest version of the algorithm we can skip the generalization on line 9 , and the generalization on line 13 of $p^{\prime \prime}$ to $p^{\prime \prime \prime}$ is obtained for free by taking $p^{\prime \prime \prime}$ to be the subset of the assumptions in $p^{\prime \prime}$ used for unsatisfiability. Finally, the strengthening of $\varphi_{A}^{\prime}$ on line 15 corresponds to $\operatorname{A.add}\left(\neg p^{\prime \prime \prime}\right)$.

We found that this (somewhat primitive) implementation already performs quite well on many instances. 


\subsection{Extensions}

For some real-life instances originating from hardware model checking problems, the basic algorithm described above often takes too many iterations to converge in reasonable time. By experimentation, we found that the following heuristics/optimizations work well for those hard cases.

Exhaustive generalization of $\boldsymbol{p}^{\prime \prime}$ to $\boldsymbol{p}^{\prime \prime \prime}$ Even though the MiniSatlike "solve under assumption" mechanism is highly successful at detecting which subset of the assumptions is important for unsatisfiability, this subset is very often far from minimal. Thus, after obtaining a reduced set from the solver's "final" conflict analysis, one can try to shrink this set further. It is natural to look for minimal ${ }^{3}$ or even minimum-sized subsets.

We implemented the following greedy approach for finding a minimal subset of the conflicting assumptions, similar to a basic destructive algorithm for MUS computations [DGHP09,Nad10,SL11]. Remove one of the assumptions - if the remaining formula is satisfiable then this assumption is deemed as necessary and must be present in all minimal assumption subsets from this point on. If the remaining formula is unsatisfiable, then the assumption is redundant and is deleted from the set of assumptions under consideration. Also note that in the case of an unsatisfiable answer, one can immediately trim the set of non-processed assumptions further (when this functionality is supported by the solver). After all of the assumptions are processed, we end up with a minimal subset as required. We refer to this approach as exhaustive B-generalization.

In general this optimization has a significant overhead on the running time of a single iteration of the loop since in the worst case it resorts to one additional SAT call for each of the assumptions in the initial set. However as we will see in the experimental section, the smaller clauses produced by this minimization are of better quality and the algorithm takes significantly fewer iterations to converge (in the same spirit as generalization of counterexamples and inductive clauses in IC3).

Forall-Exists generalization of $\boldsymbol{p}^{\prime}$ to $\boldsymbol{p}^{\prime \prime}$ Turning to generalization of $p^{\prime}$ with respect to $\varphi_{A}$ (on line 9 ), several methods have been proposed in earlier works in the context of finding all satisfying assignments to a formula (see for example [JS05]) or existential quantification (e.g. [BKK11] and [GM12]).

\footnotetext{
${ }^{3}$ I.e. the formula would become satisfiable if any of the assumptions were dropped.
} 
We implemented several variations, based on [BKK11]. We apply the following "dual-rail" construction. For each of the common variables $v \in V_{A \cap B}$, we introduce two additional fresh variables $v^{+}$and $v^{-}$, and we replace each occurrence of $v$ in $\varphi_{A}$ by $v^{+}$, and each occurrence of $\neg v$ in $\varphi_{A}$ by $v^{-}$. In addition, we add the binary clause $\left(\neg v^{+}, \neg v^{-}\right)$to prevent the solver from assigning both $v^{+}$and $v^{-}$to true. In the simpler variant to which we refer as trivial A-generalization we create the cube $p^{\prime \prime}$ from the model $p$ of $\varphi_{A}^{\prime}$ by including the literals $v$ for which $v^{+}$is set to true, and the literals $\neg v$ for which $v^{-}$is set to true. In the more complicated variants, we seek the shortest possible cubes $p^{\prime \prime}$. To this end, we create additional variables $v^{ \pm}=v^{+} \wedge v^{-}$for $v \in V_{A \cap B}$, and put a sequential counter construction [Sin05] on top of $v^{ \pm}$. This allows (passing additional assumptions to the $A$-solver) to look for cubes in $A$ which are of size at most $k$, for any given $k$, and one can find a shortest cube by setting increasing values to $k=1,2, \ldots$ By experimenting with various parameter settings, we limit the maximum value of the counter to $\min \left(15,\left|V_{A \cap B}\right|\right)$ and we do a binary search to find the minimal $k \in[1 . .15]$ if it exists. In other words we are guaranteed to end up with a shortest cube whenever it has length at most 15, and otherwise we resort to the trivial A-generalization from above. We refer to this version as counter-based A-generalization.

In general, the dual-rail construction has a negligible overhead, but searching for a shortest cube is expensive, both due to the extra logic pertaining to the sequential counter construction and the increased number of SAT-calls.

Exchanging roles of the two solvers In certain cases $\varphi_{B}$ has fewer satisfying assignments than $\varphi_{A}$ or it is easier to enumerate them. Then it might be easier to solve the "dual" problem first: compute $\varphi_{J}$ - interpolant for $\left(\varphi_{B}, \varphi_{A}\right)$, and then set $\varphi_{I} \leftarrow \neg \varphi_{J}$. This way the final interpolant is in CNF, but in most applications the precise form of the interpolant is not important (in case it is, see [BKK11] for an efficient method to convert between the two forms).

Exchanging clauses of common variables One can consider a general algorithm which uses the partitioning of $\varphi_{A} \wedge \varphi_{B}$ into $\left(\varphi_{A}, \varphi_{B}\right)$ and allows to exchange learned clauses between the two solvers as long as they consist of common variables only. In particular, such an algorithm might use a scenario when the roles of the two solvers are switched periodically, or a scenario with the two solvers running in parallel, each producing 
satisfying assignments and/or blocking the satisfying assignments found by the other solver.

Of course, if the clauses are passed from $\varphi_{A}$ to $\varphi_{B}$ and back freely, more care should be taken when assembling the final interpolant. Luckily this is not too hard due to the following (here $\varphi_{G}$ and $\varphi_{H}$ correspond to (sets of) clauses learnt from $\varphi_{A}$ and $\varphi_{B}$ respectively).

Claim 2 Suppose that $\varphi_{A} \Rightarrow \varphi_{G}$ and $\operatorname{Vars}\left(\varphi_{G}\right) \subseteq V_{A \cap B}$. Then an interpolant $\varphi_{I}=\operatorname{Itp}\left(\varphi_{A}, \varphi_{B}\right)$ can be computed as $\varphi_{I}=\operatorname{Itp}\left(\varphi_{A}, \varphi_{B} \wedge \varphi_{G}\right) \wedge \varphi_{G}$.

Proof. Let $\varphi_{J}=\operatorname{Itp}\left(\varphi_{A}, \varphi_{B} \wedge \varphi_{G}\right)$. By definition, $\varphi_{A} \Rightarrow \varphi_{J}$ and $\varphi_{A} \Rightarrow \varphi_{G}$, hence $\varphi_{A} \Rightarrow \varphi_{I}$. Also, $\varphi_{J}$ is disjoint from $\varphi_{B} \wedge \varphi_{G}$, hence $\varphi_{J} \wedge \varphi_{G}$ is disjoint from $\varphi_{B}$.

Claim 3 Suppose that $\varphi_{B} \Rightarrow \varphi_{H}$ and $\operatorname{Vars}\left(\varphi_{H}\right) \subseteq V_{A \cap B}$. Then an interpolant $\varphi_{I}=\operatorname{Itp}\left(\varphi_{A}, \varphi_{B}\right)$ can be computed as $\varphi_{I}=\operatorname{Itp}\left(\varphi_{A} \wedge \varphi_{H}, \varphi_{B}\right) \vee$ $\neg \varphi_{H}$.

Proof. Let $\varphi_{J}=\operatorname{Itp}\left(\varphi_{A} \wedge \varphi_{H}, \varphi_{B}\right) \cdot \varphi_{A} \wedge \varphi_{H} \Rightarrow \varphi_{J}$, hence $\varphi_{A} \Rightarrow \varphi_{J} \vee$ $\neg \varphi_{H}=\varphi_{I}$. Also, since both $\varphi_{J}$ and $\neg \varphi_{H}$ are disjoint from $\varphi_{B}$, so is $\varphi_{I}$.

We can keep track of the sets of clauses passed from $\varphi_{A}$ to $\varphi_{B}$ and vice versa and to reconstruct the interpolant by following the two rules above. This procedure leads to more general definitions of interpolants (not only CNF or DNF). However, if the only clauses passed from $\varphi_{A}$ to $\varphi_{B}$ are unit clauses, then the interpolant can be still computed in DNF as $\left(\vee C_{i}\right) \wedge(x)=\vee\left(C_{i} \wedge x\right)$ by distributivity.

Remark 1. Note that the blocking clauses $\neg p^{\prime \prime \prime}$ of Algorithm 1 are implied by $\varphi_{B}$, and thus can be viewed as clauses passed from $\varphi_{B}$ to $\varphi_{A}$. In other words, Algorithm 1 can be seen as employing the incremental interpolant computation dictated by Claim 3 only.

We implemented a variant of this technique which periodically instructs the $A$-solver to look for unit clauses of common variables (by running the $A$-solver with a small time-limit and a small backtrack-limit) and passing these clauses to the $B$-solver. In many cases we saw a big reduction in the total number of iterations (in several cases passing unit clauses from $\varphi_{A}$ to $\varphi_{B}$ made $\varphi_{B}$ directly unsatisfiable). The cons of this technique is that not all of the units passed from $\varphi_{A}$ to $\varphi_{B}$ are really required for unsatisfiability, while our construction adds all of these units into the interpolant. We refer to this optimization as flp (since it is reminiscent of failed literal probing in SAT-solving). 
Interpolant Strength We make a theoretical digression. In the discussion above we strove to generalize $p^{\prime}$ as much as possible, that is to describe the largest set in the projection. The motivation for this is clear - a smaller blocking clause can potentially block more points of $\varphi_{A}^{\prime}$, thus allowing the algorithm to converge faster. However for various applications the loosest possible interpolant might not be good, and it could be helpful to compute the largest blocking clause which blocks the same points in $\varphi_{A}^{\prime}$ as $\neg p^{\prime \prime \prime}$. In other words, we can seek for a subcube $q$ with $p^{\prime} \subseteq q \subseteq p^{\prime \prime \prime}$ and $\varphi_{A}^{\prime} \wedge \neg q=\varphi_{A}^{\prime} \wedge \neg p^{\prime \prime \prime}$. After such subcube $q$ is found, we can modify the line 14 of the algorithm to include $q$ instead.

We illustrate this on an example. Suppose $\left\{x_{1}, x_{2}, x_{3}, x_{4}, x_{5}\right\} \subset V_{A \cap B}$ is a subset of common variables, $p^{\prime \prime}=x_{1} \wedge x_{2} \wedge x_{3} \wedge x_{4} \wedge x_{5}$, and $p^{\prime \prime \prime}=x_{1} \wedge x_{2}$. Suppose further that $p^{\prime \prime \prime} \wedge P_{A \cap B}^{A^{\prime}}$ consists of the three points $(1,1,1,1,1, \cdot)$, $(1,1,1,0,1, \cdot),(1,1,0,1,1, \cdot)$, where $\cdot$ represents the remaining variables in $V_{A \cap B}$. Note that the variable $x_{5}$ takes the same value on $P_{A \cap B}^{A^{\prime}}$ and thus we can use $q=x_{1} \wedge x_{2} \wedge x_{5}$ instead of $x_{1} \wedge x_{2}$ in the interpolant.

Finding the maximal set of variables which are constant on $p^{\prime \prime \prime} \wedge \varphi_{A}^{\prime}$ can be done in at most $\left|p^{\prime \prime}\right|-\left|p^{\prime \prime \prime}\right|$ SAT calls. We illustrate this procedure on our example. We ask the SAT solver whether $\varphi_{A}^{\prime} \wedge x_{1} \wedge x_{2} \wedge \neg\left(x_{3} \wedge x_{4} \wedge x_{5}\right)$ is satisfiable, that is we are looking for a point in $p^{\prime \prime \prime} \wedge P_{A \cap B}^{A^{\prime}}$ with at least one different value out of $\left\{x_{3}=1, x_{4}=1, x_{5}=1\right\}$. Let's say that the solver returns the point $(1,1,1,0,1, \cdot)$ which means that the variable $x_{4}$ is not constant on $p^{\prime \prime \prime} \wedge P_{A \cap B}^{A^{\prime}}$. We refine the query asking whether $\varphi_{A} \wedge x_{1} \wedge x_{2} \wedge \neg\left(x_{3} \wedge x_{5}\right)$ is satisfiable. Now the solver returns the point $(1,1,0,1,1, \cdot)$ which means that the variable $x_{3}$ is also not constant on $p^{\prime \prime \prime} \wedge P_{A \cap B}^{A^{\prime}}$. Finally the query $\varphi_{A} \wedge x_{1} \wedge x_{2} \wedge \neg x_{5}$ is unsatisfiable, and the remaining set of variables are constant on $p^{\prime \prime \prime} \wedge P_{A \cap B}^{A^{\prime}}$.

\section{Experiments}

Before discussing concrete experimental results, let us think when we expect the suggested approach to succeed. As mentioned before, Algorithm 1 and its variations might perform well if enumerating all satisfying assignments of $\varphi_{A}$ (or $\varphi_{B}$ ) is not too hard, or whenever there exists a successful partition-based algorithm for solving $\varphi_{A} \wedge \varphi_{B}$. In particular we expect our algorithm to be successful for simple formulas with a small number $\left|V_{A \cap B}\right|$ of common variables ${ }^{4}$.

\footnotetext{
${ }^{4}$ In the worst case, all interpolants (expressed in terms of $V_{A \cap B}$ ) might be of size exponential in $\left|V_{A \cap B}\right|$ : consider formulas $\varphi_{A}, \varphi_{B}$ which enforce the parity of assignments to $V_{A \cap B}$ to be even and odd, respectively.
} 
We have evaluated our algorithm on the 465 single-property benchmarks used in the 2011 Hardware Model Checking Competition. For each of these benchmarks we unrolled the design for 11 cycles to represent the bounded model checking formula $J\left(x_{0}\right) \wedge T\left(x_{0}, x_{1}\right) \wedge \bigwedge_{i=1}^{10} T\left(x_{i}, x_{i+1}\right) \wedge$ $\bigvee_{i=1}^{11} \neg P\left(x_{i}\right)$, where $J, T$, and $P$ denote respectively the initial states of the design, the transition relation and the property being verified (see [McM03] for details). We define $\varphi^{5}=\bigwedge_{i=1}^{10} T\left(x_{i}, x_{i+1}\right) \wedge \bigvee_{i=1}^{11} \neg P\left(x_{i}\right)$, $\varphi_{B}=J\left(x_{0}\right) \wedge T\left(x_{0}, x_{1}\right)$, and cnf-ize these propositional formulas using a variant of the approach described in [CMV09]. As the underlying SAT solver we use Mage, an IBM SAT solver which supports both the incremental interface of MiniSat and the ability to compute interpolants from proofs. In all of the experiments, the time-limit was set to 1800 seconds.

In the following tables we compare the performance of standard interpolation (building an interpolant from the proof of unsatisfiability of $\varphi_{A} \wedge \varphi_{B}$, in this and only in this case the proof generation capabilities of the solver are turned on) and various schemes based on Algorithm 1. We distinguish between three versions of generalization with respect to $\varphi_{A}$ : no generalization at all (no A-gen), trivial A-generalization (triv A-gen) and counter-based A-generalization (cntr A-gen). The latter two versions are described in Section 3.3 and require dual-rail encoding. We also consider three versions of generalization with respect to $\varphi_{B}$ : no generalization at all (no B-gen), the generalization based on the conflicting assumptions returned by the solver (std B-gen) and exhaustive B-generalization from Section 3.3 (exh B-gen). The results are summarized in Table 1. The second, third and fourth columns respectively denote the numbers of satisfied, unsatisfied and time-out instances, and the last column denotes the total time of the run. First of all, we see that generalizing with respect to $B$ is crucial and that counter-based A-generalization is mostly unhelpful (in fact, even the version of $A$-generalization with the unrestricted counter size on average only removes at most $5 \%-10 \%$ of the literals of $p^{\prime}$, while the exhaustive $B$-generalization usually removes $90 \%$ of the literals and more). We also note that the standard interpolation performs best in terms of time (however, there was in fact one testcase where the version (no A-gen, std B-gen) finished in 1184 seconds, while the standard interpolation timed out). It should be noted that in this and subsequent experiments all the observed phenomena are consistent across individual instances (and not only in the bulk).

We omit the inferior configurations and restrict to the test cases on which each configuration returned with a SAT or UNSAT answer - there

\footnotetext{
${ }^{5}$ The benefits of this "opposite" splitting are discussed later.
} 


\begin{tabular}{l|c|c|c|c} 
Variant & SAT & UNSAT & TO & Total Running Time (s) \\
\hline standard interpolation & 14 & 429 & 22 & 49,153 \\
no A-gen, no B-gen & 1 & 22 & 442 & 767,678 \\
no A-gen, std B-gen & 14 & 420 & 31 & 79,686 \\
no A-gen, exh B-gen & 14 & 421 & 30 & 79,754 \\
triv A-gen, no B-gen & 1 & 25 & 439 & 762,135 \\
triv A-gen, std B-gen & 13 & 419 & 33 & 83,759 \\
triv A-gen, exh B-gen & 14 & 420 & 31 & 79,599 \\
cntr A-gen, no B-gen & 1 & 17 & 447 & 783,311 \\
cntr A-gen, std B-gen & 10 & 393 & 62 & 124,170 \\
cntr A-gen, exh B-gen & 10 & 399 & 56 & 115,312
\end{tabular}

Table 1. Comparison of runtimes on 465 single property benchmarks from HWMCC11

are 430 such configurations. The comparison of the total number of iterations (column 2), the total interpolant size (column 3) and the total running time (column 4) are provided in Table 2. For the standard interpolation the number of iterations is meaningless and the size represents the number of gates in the non-optimized circuits (i.e. no structural hashing, etc. has been performed). For the remaining configurations the size represents the total number of literals in the computed interpolants (in $\mathrm{CNF}$ ). Even though it is clear that the 4 schemes based on Algorithm 1 are on average 7 times slower than standard interpolation, it is interesting to note that they produce interpolants of much smaller size (up to 900 times). In particular, they require no need for further minimization. Next, it seems that on our test cases the extra time spent by a round of exhaustive generalization is compensated by fewer iterations required for the algorithm to converge. Finally, the dual-rail encoding and the trivial version A-generalizations seem to have a small positive impact on the size of the interpolant.

\begin{tabular}{l|c|c|c} 
Variant & Total \#iters & Total itp size & Total Running Time (s) \\
\hline standard interpolation & 0 & $90,922,242$ & 3,842 \\
no A-gen, std B-gen & 129,506 & 837,273 & 19,138 \\
no A-gen, exh B-gen & 80,764 & 119,975 & 22,603 \\
triv A-gen, std B-gen & 133,903 & 857,081 & 22,286 \\
triv A-gen, exh B-gen & 77,752 & 117,105 & 22,637
\end{tabular}

Table 2. Comparison of numbers of iterations and interpolant sizes on 430 benchmarks

We have performed an additional experiment to see the value of periodically passing unit clauses from $\varphi_{A}$ to $\varphi_{B}$ (the flp technique described in 
Section 3.3). To this end we compare the (no A-gen, exh B-gen) configuration with a version of itself, where at the start and every 100 iterations the $A$-solver is instructed to look for unit clauses of common variables and to pass them to the $B$-solver. The results are summarized in Table 3 . As usual, we restrict only to the benchmarks where both versions complete - there are 407 such test cases. The interpolant size now measures the total number of literals in all the passed clauses (this corresponds to the previous definition when flp is disabled, and includes the number of unit clauses when flp is enabled). Activation of flp increases the running times (and the number of time-outs), but reduces the total number of iterations by about 4 times. On the other hand, most of the unit clauses detected during flp are irrelevant for the algorithm, and they increase the interpolant size.

\begin{tabular}{l|c|c|c} 
Variant & Total \#iters & Total itp size & Total Running Time (s) \\
\hline no A-gen, exh B-gen & 73,071 & 147,358 & 15,532 \\
no A-gen, exh B-gen, flp & 18,685 & 184,619 & 43,374
\end{tabular}

Table 3. Measuring the effect of flp on 407 benchmarks

A couple of additional remarks are in order. First, the size of the final interpolant can serve as a rough estimate for the total memory consumption of an algorithm. Second, in our experience enabling proof-logging techniques for the standard interpolation takes a very small overhead (around 5\%), while the overhead of recording blocking (or more generally exchanged) clauses is absolutely negligible. Thus, the running times really represent a comparison between showing unsatisfiability of $\varphi_{A} \wedge \varphi_{B}$ using a single call to a SAT-solver and using various variants of a partitionbased algorithm. Finally, these experiments should be taken only as a proof of concept of the methods presented. In fact, the current setup benefits our approach in two ways: 1) $\varphi_{B}$ is a more restricted formula and so potentially has less satisfying assignments than $\varphi_{A}$, and 2) $\varphi_{A}$ is a much simpler formula and so potentially allows for shorter explanation of the inconsistency between a satisfying assignment to $\varphi_{B}$ and $\varphi_{A}$, that is for shorter cubes $p^{\prime \prime \prime}$. Indeed, the experiments with roles of $\varphi_{A}$ and $\varphi_{B}$ reversed resulted in inferior performance (nearly on all instances).

\section{Conclusions and Future Work}

We described an incremental algorithm for computing Craig interpolants for a pair of mutually unsatisfiable formulas. The most significant advantage of this algorithm is its simplicity - it does not depend on the 
underlying solver's ability to produce refutations and thus can be quickly implemented on top of any SAT-solver. In particular, it has the advantage of immediately benefiting from rapid improvements of modern SAT solvers which do not produce proofs.

At this stage, the main contribution of this work is theoretical, rather than practical. We have observed the need for better partition-based algorithms. We have suggested several heuristics towards this goal, but the experimental results show inferior performance (in terms of runtime) compared to a single monolithic call. If more efficient partition-based algorithms are discovered, this work shows how an interpolant may be easily and efficiently reconstructed afterwards. We have also described a technique to vary the strength of the computed interpolant.

On ther other hand, our algorithms are much lighter in terms of memory consumption (even though the size of a proof is linear in the running time of the solver, such proofs are usually huge), and as seen in experiments, the sizes of the interpolants produced are several orders of magnitude smaller than the sizes of the interpolants constructed from proofs. With this in mind (and in the spirit of [PG00]), we can view the algorithm as the last resort for computing interpolants, when all of the conventional techniques have failed.

One especially interesting direction for further study is to see how much the proposed technique for computing interpolants can be used inside the original interpolation algorithm for model checking [McM03]. The source of inspiration for this is the success of the IC3 technique [Bra11], which shows that it is often possible to efficiently characterize an over-approximation to states reachable within a certain number of cycles as a conjunction of clauses defined on state-variables only. Note that by splitting the bounded model checking formula as we described - with $\varphi_{A}=\bigwedge_{i=1}^{k} T\left(x_{i}, x_{i+1}\right) \wedge \bigvee_{i=1}^{k+1} \neg P\left(x_{i}\right)$, and $\varphi_{B}=J\left(x_{0}\right) \wedge T\left(x_{0}, x_{1}\right)$, the interpolant $\varphi_{I}$ for $\left(\varphi_{A}, \varphi_{B}\right)$ is computed as a DNF, and hence $\neg \varphi_{A}$ representing an over-approximation of states reachable in one step is precisely in CNF form.

\section{References}

[ANORC10] Roberto Javier Asín Achá, Robert Nieuwenhuis, Albert Oliveras, and Enric Rodríguez-Carbonell. Practical algorithms for unsatisfiability proof and core generation in SAT solvers. AI Commun., 23(2-3):145-157, 2010.

[BKK11] Jörg Brauer, Andy King, and Jael Kriener. Existential quantification as incremental SAT. In $C A V$, pages 191-207, 2011.

[Bra11] Aaron R. Bradley. SAT-based model checking without unrolling. In $V M$ CAI, pages 70-87, 2011. 
[CMV09] Benjamin Chambers, Panagiotis Manolios, and Daron Vroon. Faster SAT solving with better CNF generation. In DATE, pages 1590-1595, 2009.

[Cra57] William Craig. Linear reasoning. a new form of the Herbrand-Gentzen theorem. J. Symb. Log., 22(3):250-268, 1957.

[DGHP09] Christian Desrosiers, Philippe Galinier, Alain Hertz, and Sandrine Paroz. Using heuristics to find minimal unsatisfiable subformulas in satisfiability problems. J. Comb. Optim., 18(2):124-150, 2009.

[ES03] N. Eén and N. Sörensson. An extensible SAT-solver. In SAT, pages 502518, 2003.

[GM12] Eugene Goldberg and Panagiotis Manolios. Quantifier elimination by dependency sequents. CoRR, abs/1201.5653, 2012.

[JS05] HoonSang Jin and Fabio Somenzi. Prime clauses for fast enumeration of satisfying assignments to Boolean circuits. In $D A C$, pages 750-753, 2005.

[Kra97] Jan Krajícek. Interpolation theorems, lower bounds for proof systems, and independence results for bounded arithmetic. J. Symb. Log., 62(2):457486, 1997.

[KW10] Daniel Kroening and Georg Weissenbacher. Verification and falsification of programs with loops using predicate abstraction. Formal Asp. Comput., 22(2):105-128, 2010.

[LJH08] Ruei-Rung Lee, Jie-Hong Roland Jiang, and Wei-Lun Hung. Bidecomposing large Boolean functions via interpolation and satisfiability solving. In $D A C$, pages 636-641, 2008.

[LJHM07] Chih-Chun Lee, Jie-Hong Roland Jiang, Chung-Yang Huang, and Alan Mishchenko. Scalable exploration of functional dependency by interpolation and incremental SAT solving. In ICCAD, pages 227-233, 2007.

[McM02] Kenneth L. McMillan. Applying SAT methods in unbounded symbolic model checking. In $C A V$, pages 250-264, 2002.

[McM03] Kenneth L. McMillan. Interpolation and SAT-based model checking. In $C A V$, pages $1-13,2003$.

[McM05] Kenneth L. McMillan. Applications of Craig interpolants in model checking. In TACAS, pages 1-12, 2005.

[McM10] Kenneth L. McMillan. Lazy annotation for program testing and verification. In $C A V$, pages 104-118, 2010.

[Nad10] Alexander Nadel. Boosting minimal unsatisfiable core extraction. In FM$C A D$, pages 221-229, 2010.

[PG00] Tai Joon Park and Allen Van Gelder. Partitioning methods for satisfiability testing on large formulas. Inf. Comput., 162(1-2):179-184, 2000.

[Pud97] Pavel Pudlák. Lower bounds for resolution and cutting plane proofs and monotone computations. J. Symb. Log., 62(3):981-998, 1997.

[Sin05] Carsten Sinz. Towards an optimal CNF encoding of Boolean cardinality constraints. In $C P$, pages $827-831,2005$.

[SL11] João P. Marques Silva and Inês Lynce. On improving MUS extraction algorithms. In SAT, pages 159-173, 2011.

[ZM03] Lintao Zhang and Sharad Malik. Validating SAT solvers using an independent resolution-based checker: Practical implementations and other applications. In DATE, pages 10880-10885, 2003. 